\title{
Vibrations aléatoires des structures Notions fondamentales
}

A. Coubat Laboratoire de mécanique appliquée associé au CNRS, Besançon

R.J. Gibert CEA - Centre d'études nucléaires de Saclay

L'exposé est une partie introductive qui se propose d'expliciter de façon élémentaire les notions de base et le vocabulaire utilisés dans le domaine des vibrations aléatoires. Ainsi sont rappelés quelques éléments de théorie des probabilités, des variables et des processus aléatoires puis sont indiqués quelques outils et méthodes d'analyse des systèmes linéaires soumis à une excitation aléatoire stationnaire.

Lorsqu'un système est soumis à des sollicitations aléatoires il est essentiel de connaitre les caractéristiques de sa réponse. Nous nous proposons de préciser les grandeurs mathématiques et le vocabulaire communément utilisés dans ce domaine dit des "vibrations aléatoires".

\section{Aperçu de théorie des probabilités}

\subsection{Les axiomes}

Lorsqu'on observe un phénomène aléatoire le résultat obtenu, inconnu par avance, appartient à l'ensemble $\Omega$ des résultats possibles. Les "événements " sont constitués par une famille $F$ de sous ensembles de $\Omega$, comprenant $\Omega$ et stable pour la réunion et la complémentation.

Un événement $A$ est dit réalisé lorsque le résultat $\omega$ effectivement obtenu à l'issue de l'expérience aléatoire appartient à $A$. Une probabilité est définie comme une application $P$ de $\mathbf{F}$ dans $\mathrm{R}$ obéissant aux axiomes :

$0 \leqslant P[A] \leqslant 1$ pour tout $A$ de $\mathrm{F}(P[A]$ constitue la probabilité de $A$ );

$P[\Omega]=1$, ( $\Omega$ est appelé événement sûr);

$P\left[\cup A_{i}\right]=\Sigma P\left[A_{i}\right]$ pour $\operatorname{des} A_{i}$ mutuellement exclusifs tels que $A_{i} \cap A_{j}=\Phi$.

\subsection{Principales conséquences}

$P[\Omega \backslash A]=1-P[A] ; \quad(\Omega \backslash A$ est le contraire de $A$; $\Phi=\Omega \backslash \Omega$ est l'événement impossible et $P[\Phi]=0$ ).

Si $\Omega$ est constitué par la réunion d'éventualités $\omega$ équiprobables on retrouve le calcul traditionnel d'une probabilité : $P[A]=n(A) / n(\Omega), n(A)$ et $n(\Omega)$ étant les nombres d'éventualités de $A$ et de $\Omega$.

\subsection{Probabilités conditionnelles}

Etant donné $B \neq \Phi$, on appelle probabilité conditionnelle de $A$ sachant $B$ le quotient: $P[A / B]=$ $P[A \cap B] / P[B]$.

Si $A$ et $B$ sont indépendants alors $P[A / B]=P[A]$ et $P[A \cap B]=P[A] P[B]$.

\subsection{Variable aléatoire}

Une v.a. $X$ est une application de $\Omega$ dans $\mathrm{R}$ telle que pour tout $x$ existe dans $\mathbf{F}$ l'événement correspondant: $\{\omega$ : $X(\omega)<x\}$. Par conséquent on peut associer une probabilité à la v.a. $X: P[X<x]=P[\{\omega: X(\omega)<x\}]$. On peut aussi trouver dans $F$ des événements tels que $\{\omega$ : $\left.x_{1} \leqslant X(\omega)<x_{2}\right\}$ ou $\{\omega: X(\omega)=x\}$ auxquels sont associées les probabilités $P\left[x_{1} \leqslant X<x_{2}\right]$ ou $P[X=x]$. Une v.a. est discrète lorsque l'ensemble des $X(\omega)=x$ possibles est fini ou infini dénombrable; elle est continue

Random vibrations of structures : fundamental notions.

This brief account is an introduction which seeks to explain in an elementary way the basic notions and the vocabulary used in the field of random vibrations. Accordingly, some theoretical elements of probabilities, random variables and random processes are considered. Next, some tools and methods for analyzing linear systems subject to a stationary random excitation will be indicated. 
lorsque $X(\omega)$ peut prendre toute valeur dans un intervalle réel non vide.

\subsection{Loi de probabilité d'une variable aléatoire}

- Pour une v.a. discrète $X$ c'est la correspondance $x_{i} \rightarrow$ $P\left[X=x_{i}\right]$. Ainsi pour la v.a. binomiale qui mesure le nombre de succès dans une suite de $n$ alternatives successives indépendantes, chacune de ces alternatives aboutissant à un succès de probabilité $p$ ou à un échec de probabilité $q=1-p$, on a : $P[X=k]=C_{n}^{k} p^{k} q^{n-k}$ avec $0 \leqslant k \leqslant n$.

- La fonction de distribution d'une v.a. $X$ est la fonction $F$ de $\mathbf{R}$ dans $[0,1]$ définie par : $x \rightarrow F(x)=P[X<x]$. Il est immédiat que : $F(-\infty)=0, F(+\infty)=1 ; F\left(x_{2}\right)-$ $F\left(x_{1}\right)=P\left[x_{1} \leqslant X<x_{2}\right] \geqslant 0$ pour $x_{1}<x_{2}$.

- La dérivée de la fonction de distribution de probabilité est la densité de probabilité $f$. Elle caractérise parfaitement une v.a. continue et l'on a :

$$
\begin{aligned}
P[a \leqslant X<b]= & \int_{a}^{b} f(x) d x ; \int_{-\infty}^{+\infty} f(x) d x=1 ; \\
& f(+\infty)=f(-\infty)=0 ; f(x) \geqslant 0 .
\end{aligned}
$$

La v.a. normale est une v.a. continue que l'on rencontre fréquemment comme résultante additive de nombreuses v.a. indépendantes, aucune n'étant prépondérante par rapport aux autres (théorème central limite). Elle est entièrement définie par deux paramètres $m$ et $\sigma$ et l'on a pour la densité :

$$
f(x)=\left(2 \pi \sigma^{2}\right)^{-1 / 2} \exp \left\{-[(x-m) / \sigma]^{2} / 2\right\} .
$$

\subsection{Variables aléatoires multidimensionnelles}

Il peut être intéressant de considérer simultanément plusieurs v.a., par exemple deux v.a. $X_{1}$ et $X_{2}$, sous l'angle de composantes d'une v.a. vectorielle $\mathbf{X}$. Les notions de distribution et de densité de probabilité se généralisent aisément. Ainsi :

$$
F_{\mathbf{X}}(x)=F_{X_{1} X_{2}}\left(x_{1}, x_{2}\right)=P\left[\left\{X_{1}<x_{1}\right\} \cap\left\{X_{2}<x_{2}\right\}\right] .
$$

Si $X_{1}$ et $X_{2}$ sont continues la densité de probabilité est:

$$
f_{\mathrm{X}}(x)=f_{X_{1} X_{2}}\left(x_{1}, x_{2}\right)=\partial^{2} F_{X_{1} X_{2}}\left[x_{1}, x_{2}\right] / \partial x_{1} \partial x_{2} .
$$

Citons quelques relations parmi de nombreuses autres :

$$
\begin{aligned}
\int_{-\infty}^{+\infty} \int_{-\infty}^{+\infty} f_{X_{1} X_{2}}\left(x_{1}, x_{2}\right) d x_{1} d x_{2} & =1 \\
f_{X_{1}}\left(x_{1}\right) & =\int_{-\infty}^{+\infty} f_{x_{1} X_{2}}\left(x_{1}, x_{2}\right) d x_{2}
\end{aligned}
$$

Si $X_{1}$ et $X_{2}$ sont indépendantes :

$$
f_{X_{1} X_{2}}\left(x_{1}, x_{1}\right)=f_{X_{1}}\left(x_{1}\right) f_{X_{2}}\left(x_{2}\right) .
$$

Sinon :

$f_{X_{1} X_{2}}\left(x_{1}, x_{2}\right)=f_{X_{2}}\left(x_{2}\right) f_{X_{1} \mid X_{2}}\left(x_{1} \mid x_{2}\right), f_{X_{1} \mid X_{2}}\left(x_{1} \mid x_{2}\right) d x_{1}$ étant la probabilité conditionnelle de trouver $X_{1}$ dans $\left(x_{1}, x_{1}+d x_{1}\right)$ sachant que $X_{2}=x_{2}$.

\subsection{Espérance mathématique}

Dans le cas d'une v.a. discrète c'est

$$
E[X]=\Sigma x_{i} P\left[X=x_{i}\right] .
$$

Dans le cas d'une v.a. continue c'est

$$
E[X]=\int_{-\infty}^{+\infty} x f(x) d x
$$

Si $Y=g\left(X_{1}, \ldots, X_{n}\right), g$ fonction de type déterministe et si l'on connaît la densité de la v.a. vectorielle $\mathbf{X}$ soit $f_{X 1 \ldots X_{n}}\left(x_{1}, \ldots, x_{n}\right)$ alors :

$$
\begin{aligned}
& E[Y]=E\left[g\left(X_{1}, \ldots, X_{n}\right)\right] \\
& =\int_{-\infty}^{+\infty} \ldots \int_{-\infty}^{+\infty} g\left(x_{1}, \ldots, x_{n}\right) f_{X 1 \ldots X n}\left(x_{1}, \ldots, x_{n}\right) d x_{1} \ldots d x_{n} .
\end{aligned}
$$

Il en résulte que les opérateurs de sommation et d'espérance sont commutatifs :

$$
E\left[\Sigma g_{i}\left(X_{i}\right)\right]=\Sigma\left(E\left[g_{i}\left(X_{i}\right)\right]\right) .
$$

En revanche: $E\left[\Pi g_{i}\left(X_{i}\right)\right]=\Pi\left(E\left[g_{i}\left(X_{i}\right)\right]\right)$ n'est vrai que si les v.a. $X_{i}$ sont indépendantes.

\subsection{Moments}

Ce sont les espérances des puissances d'une ou plusieurs v.a. : $E\left[X^{n}\right]$ est le $n^{\text {e }}$ moment de $X, E\left[(X-E[X])^{n}\right]$ est le $n^{e}$ moment centré de $X, E\left[(X-E[X])^{m}(Y-E[Y])^{n}\right]$ est le $(m+n)^{\mathrm{e}}$ moment croisé centré de $X$ et $Y$. Les cas particuliers importants sont la variance: $\operatorname{Var}(X)=E\left[(X-E[X])^{2}\right]=E\left[X^{2}\right]-(E[X])^{2}$ (dont la racine carrée $\sigma_{X}$ est l'écart-type) et le moment croisé d'ordre $1+1$ de $X$ et $Y$, appelé covariance :

$$
\begin{aligned}
\operatorname{Cov}(X, Y)=E[(X-E[X]) & (Y-E[Y])] \\
= & E[(X-E[X])(Y-E[Y])] .
\end{aligned}
$$

L'espérance mathématique $E[X]$ représente une valeur moyenne de la v.a. tandis que l'écart-type $\sigma$ caractérise la dispersion des valeurs autour de $E[X]$ comme le montre l'inégalité de Bienaymé-Tchébychev:

$P[E[X]-k \sigma<X<E[X]+k \sigma] \geqslant 1-(1 / k)^{2}$.

Le quotient $r=\operatorname{Cov}(X, Y) / \sigma_{X} \sigma_{Y}$ est appelé coefficient de corrélation des v.a. $X$ et $Y$. On a : $-1 \leqslant r \leqslant 1$. Si les v.a. sont indépendantes $r=0$, si elles sont liées linéairement $|r|=1$.

Si $X_{1}, \ldots, X_{n}$ sont indépendantes : $\operatorname{Var}\left(X_{1}+\ldots+X_{n}\right)=$ $\operatorname{Var}\left(X_{1}\right)+\ldots+\operatorname{Var}\left(X_{n}\right)$.

\subsection{Exemples}

Pour la v.a. binomiale: $E[X]=n p$ et $\operatorname{Var}(X)=$ $n p(1-p)$ tandis que pour la v.a. normale $E[X]=m$ et $\operatorname{Var}(X)=\sigma^{2}$. 


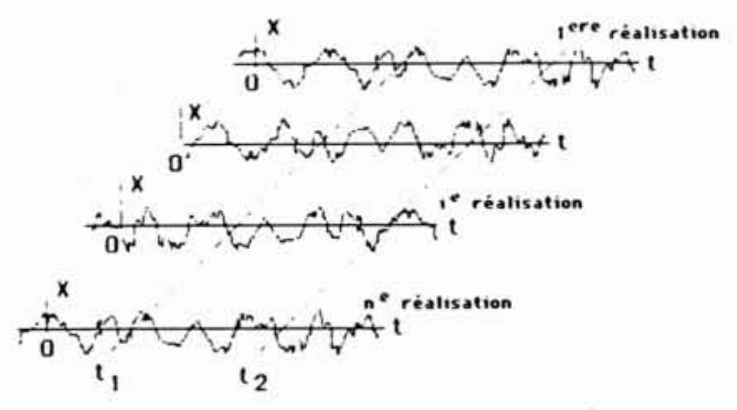

1 . Processus aléatoire.

\section{Processus aléatoires}

\subsection{Définition}

Lorsqu'on observe plusieurs réalisations de la grandeur $X$ décrite par un processus aléatoire on obtient des enregistrements différents (figure 1). Pour chacune des réalisations la connaissance de la valeur observée au temps $t_{1}$ ne permet pas de prévoir la valeur au temps $t_{2}$. Ainsi on appelle processus ou fonction aléatoire un ensemble de variables aléatoires $X(t)$, indicées par le temps $t \in[0, T] . X\left(t_{1}\right)$ et $X\left(t_{2}\right)$ sont donc deux v.a. dont les valeurs possibles apparaissent sur chacune des réalisations aux temps $t_{1}$ et $t_{2}$. Le processus est noté $\{X(t)\}$ ou parfois simplement $X(t)$.

\subsection{Caractérisation}

Pour bien spécifier un processus aléatoire $\{X(t)\}$, il faudrait connaître la densité de probabilité d'ordre $n$ quelconque : $p_{|X|}\left(x_{1}, t_{1} ; \ldots ; x_{n}, t_{n}\right)$ qui est une fonction des $t_{i}$ et $x_{i}$ et telle que $p_{\{X\}} d x_{1} \ldots d x_{n}$ représente la probabilité de trouver les $X\left(t_{i}\right)$ dans les intervalles $\left(x_{i}, x_{i}+d x_{i}\right)$.

La connaissance d'une densité d'ordre $n$ entraîne celle d'une densité d'ordre inférieur par intégration :

$$
\begin{aligned}
\int \ldots \int p\{X\}\left(x_{1}, t_{1} ; x_{2}, t_{2} ; \ldots ; x_{n+k}, t_{n+k}\right) & d x_{n+1} \ldots d x_{n+k} \\
= & p_{\{X\}}\left(x_{1}, t_{1} ; \ldots ; x_{n}, t_{n}\right) .
\end{aligned}
$$

On se contente souvent des densités d'ordre 2 : elles permettent de déduire les fonctions moments importantes pour les applications :

- la fonction moyenne: $E[X(t)]=\mu_{X}(t)$,

- la fonction d'autocorrélation: $E\left[X\left(t_{1}\right) X\left(t_{2}\right)\right]=$ $\Phi_{X X}\left(t_{1}, t_{2}\right)$

- la fonction d'intercorrélation si on considère deux processus différents $\{X(t)\},\{Y(t)\}$ :

$\Phi_{X Y}\left(t_{1}, t_{2}\right)=E\left[X\left(t_{1}\right) Y\left(t_{2}\right)\right]$.

- la fonction d'autocovariance :

$K_{X X}\left(t_{1}, t_{2}\right)=E\left[\left(X\left(t_{1}\right)-\mu_{X}\left(t_{1}\right)\right)\left(X\left(t_{2}\right)-\mu_{X}\left(t_{2}\right)\right)\right]$ qui devient la fonction variance pour $t_{1}=t_{2}$.

\subsection{Processus stationnaires}

Un processus est stationnaire quand la structure de sa densité de probabilité d'ordre $n$ quelconque n'est pas modifiée par un changement d'origine des temps :

$$
p_{\{X\}}\left(x_{1}, t_{1}, \ldots, x_{n}, t_{n}\right)=p_{\{X\}}\left(x_{1}, t_{1}+a ; \ldots ; x_{n}, t_{n}+a\right) .
$$

Dans la pratique, on s'intéresse aux processus faiblement stationnaires où seules les densités d'ordre 1 et 2 vérifient l'égalité précédente.

Alors les moments d'ordre 1 et 2 ne sont pas affectés par un changement d'origine des temps. Ainsi $E\left[X^{k}(t)\right]$ est indépendant de $t, E\left[X^{k}\left(t_{1}\right) X^{1}\left(t_{2}\right)\right]$ dépend seulement de la différence : $\tau=t_{2}-t_{1}$. Les grandeurs les plus importantes sont la moyenne $E[X(t)]=\mu_{X}$ (qui est donc constante) et la fonction d'autocorrélation: $\Phi_{X X}\left(t_{1}, t_{2}\right)=$ $R_{X X}(\tau)$ avec $\tau=t_{1}-t_{2}$

Il est immédiat que $R_{X X}(\tau)=R_{X X}(-\tau)$ et que $R_{X X}(0)=E\left[X^{2}(t)\right]$.

Pour deux processus $\{X(t)\},\{Y(t)\}$ conjointement stationnaires, les notions sont analogues. Ainsi $\Phi_{X Y}\left(t_{1}, t_{2}\right)=R_{X Y}(\tau)=R_{Y X}(-\tau)$.

On montre que : $\left|R_{X Y}(\tau)\right| \leqslant\left(R_{X X}(0) R_{Y Y}(0)\right)^{1 / 2}$ d'où $\left|R_{X X}(\tau)\right| \leqslant R_{X X}(0)$.

Une grandeur joue un rôle très important dans l'analyse des processus stationnaires: la fonction de densité spectrale de puissance (DSP) $S_{X X}(\omega)$, transformée de Fourier de $R_{X X}(\tau)$ :

$$
S_{X X}(\omega)=(1 / 2 \pi) \int_{-\infty}^{+\infty} R_{X X}(\tau) \exp (-i \omega \tau) d \tau .
$$

Par transformée inverse de Fourier :

$$
R_{X X}(\tau)=\int_{-\infty}^{+\infty} S_{X X}(\omega) \exp (i \omega \tau) d \omega .
$$

De cette relation découle :

$$
E\left[X^{2}(t)\right]=R_{X X}(0)=\int_{-\infty}^{+\infty} S_{X X}(\omega) d \omega
$$

qui donne l'interprétation physique de $S_{X X}(\omega)$.

Le "bruit blanc " est un processus théorique, idéalisation d'un processus stochastique à large bande, pour lequel $S_{X X}(\omega)=S_{0}=$ constante.

\subsection{Processus ergodiques}

Ce sont des processus stationnaires pour lesquels chaque réalisation considérée sur un temps $T$ suffisamment grand est représentative de l'ensemble des réalisations. Toutes les moyennes des divers ordres se déduisent par des moyennes temporelles sur une seule réalisation :

$$
\begin{aligned}
\mu_{X}=(1 / T) & \int_{0}^{T} X(t) d t \\
& R_{X X}(\tau)=(T-\tau)^{-1} \int_{0}^{T} X(t) X(t+\tau) d t .
\end{aligned}
$$

Dans la plupart des travaux, l'ergodicité est supposée a priori. 


\subsection{Processus gaussiens}

Le processus est alors constitué d'une famille de v.a. $X(t)$ gaussiennes $\mathbf{N}(\mu(t), \sigma(t))$. Pour $t_{1} \neq t_{2}$ les v.a. $X\left(t_{1}\right)$ et $X\left(t_{2}\right)$ sont conjointement normales c'est-à-dire que la densité de probabilité de $X\left(t_{1}\right), X\left(t_{2}\right)$ s'écrit en posant :

$\sigma_{i}=\sigma\left(t_{i}\right) ; \mu_{i}=\mu\left(t_{i}\right) ; \rho=\operatorname{Cov}\left(X\left(t_{1}\right), X\left(t_{2}\right) / \sigma_{1} \sigma_{2}:\right.$

$p_{X 1 X 2}\left(x_{1}, t_{1} ; x_{2}, t_{2}\right)=$

$$
\left(2 \pi \sigma_{1} \sigma_{2}\left(1-\rho^{2}\right)^{1 / 2}\right)^{-1} \exp \left\{-f /\left(2 \sigma_{1}^{2} \sigma_{2}^{2}\left(1-\rho^{2}\right)\right)\right\}
$$

où $f$ est donné par:

$$
\begin{aligned}
f=\sigma_{2}^{2}\left(x_{1}-\mu_{1}\right)^{2}-2 \rho\left(x_{1}-\right. & \left.\mu_{1}\right) \\
& \left(x_{2}-\mu_{2}\right)+\sigma_{1}^{2}\left(x_{2}-\mu_{2}\right)^{2} .
\end{aligned}
$$

\subsection{Processus de Markov}

Ce sont des processus dont l'évolution de la densité de probabilité conditionnelle dépend du passé le plus récent c'est-à-dire :

$$
p_{\{X \mid}\left(x_{n}, t_{n} \mid x_{n-1}, t_{n-1} ; \ldots ; x_{1}, t_{1}\right)=p_{\{X\}}\left(x_{n}, t_{n} \mid x_{n-1}, t_{n-1}\right)
$$

avec $t_{1}<t_{2}<\ldots<t_{n}$.

La densité intervenant au second membre est la densité de transition, habituellement notée $q_{|X|}\left(x, t \mid x_{0}, t_{0}\right)$ avec $t>t_{0}$.

Un exemple en est donné par la fonction aléatoire de Wiener: $\{W(t) ; t>0\}$ qui possède les propriétés suivantes :

$W(0)=0 ; \quad E[W(t)]=0 ; \quad E\left[W\left(t_{1}\right) W\left(\mathrm{t}_{2}\right)\right]=2 D$ $\inf \left(t_{1}, t_{2}\right)$ avec $D>0$.

La densité de probabilité est gaussienne: $(4 \pi D t)^{-1 / 2}$ $\exp \left\{-w^{2} / 4 D t\right\}$.

La dérivée de ce processus, au sens des distributions, est le bruit blanc de densité spectrale de puissance $2 D$.

L'importance des processus de Markov provient du fait que la densité de transition satisfait l'équation différentielle dite de Fokker-Planck-Kolmogorov, laquelle s'écrit dans le cas d'un processus scalaire :

$$
\partial q / \partial t=\Sigma\left[(-1)^{n} / n !\right] \partial^{n}\left[A_{n} q\right] / \partial x^{n},
$$

où $n \in \mathrm{N}^{\star}$, où $A_{n}$ est la limite de

$$
(\Delta t)^{-1} E\left[(X(t+\Delta t)-X(t))^{n} \mid X(t)=x\right]
$$

lorsque $\Delta t \rightarrow 0$.

En général les $A_{n}$ sont déduits des équations qui régissent le comportement du système et sont nuls pour $n \geqslant 3$.

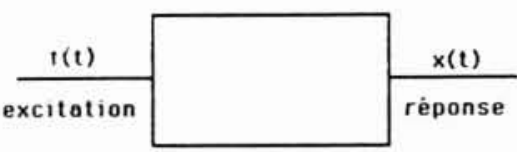

2. Modèle linéaire.
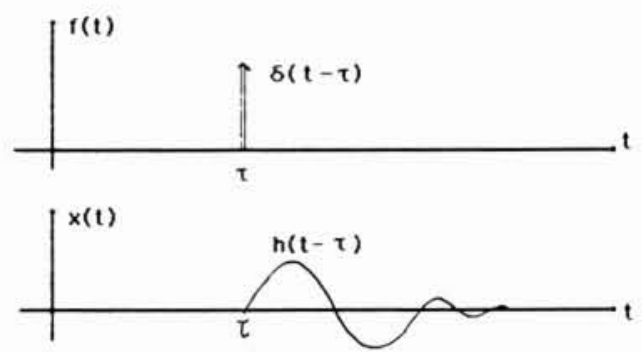

3. Réponse à l'impulsion de Dirac.

\section{Réponse des systèmes linéaires à une excitation aléatoire stationnaire}

\subsection{Systèmes dynamiques à un degré de liberté (figure 2)}

\subsubsection{Problème " unitaire" dans le temps}

Soit $h(t)$ la réponse à l'impulsion de Dirac $\delta(t)$ (figure 3). Alors, par superposition, la réponse à une excitation $f(t)$ quelconque est :

$x(t)=\int_{-\infty}^{+\infty} f(\tau) h(t-\tau) d \tau$

$$
=\int_{-\infty}^{+\infty} h(\theta) f(t-\theta) d \theta .
$$

Par exemple pour le système masse-ressort-dash-pot décrit par l'équation :

$$
d^{2} x / d t^{2}+2 a \omega_{0} d x / d t+\omega_{0}^{2} x=f(t),
$$

on a $h(t)=0$ pour $t<0$ et

$h(t)=\left(\omega_{0} \sqrt{1-a^{2}} e^{\omega \omega 0} t\right)^{-1} \sin \left(\sqrt{1-a^{2}} \omega_{0} t\right)$

pour $t \geqslant 0$.

\subsubsection{Problème " unitaire " en fréquence}

La réponse à l'excitation harmonique $e^{i \omega t}$ est $H(\omega) e^{i \omega t}$. $H(\omega)$ est la fonction de transfert du système, liée à la réponse impulsionnelle par transformation de Fourier:

$$
H(\omega)=\int_{-\infty}^{+\infty} h(t) e^{-i \omega t} d t .
$$

La réponse à une excitation $f(t)$ quelconque envisagée comme la somme :

$$
f(t)=(2 \pi)^{-1} \int_{-\infty}^{+\infty} F(\omega) e^{i \omega t} d \omega
$$

s'écrit, compte tenu du principe de superposition :

$$
x(t)=(2 \pi)^{-1} \int_{-\infty}^{+\infty} H(\omega) F(\omega) e^{i \omega t} d \omega .
$$


En considérant la décomposition spectrale de $x(t)$ :

$$
x(t)=(2 \pi)^{-1} \int_{-\infty}^{+\infty} X(\omega) e^{i \omega t} d \omega,
$$

il résulte :

$$
X(\omega)=F(\omega) H(\omega) .
$$

Pour l'exemple envisagé :

$$
H(\omega)=\left(\omega_{0}^{2}-\omega^{2}+2 i a \omega_{0} \omega\right)^{-1} .
$$

\subsubsection{Statistiques de réponse}

- Les moyennes d'entrée et de sortie du système sont proportionnelles :

$$
\begin{aligned}
E[X(t)]=\mu_{x}=\int_{-\infty}^{+\infty} & h(\theta) E[f(t-\theta)] d \theta \\
& =\mu_{f} \int_{-\infty}^{+\infty} h(\theta) d \theta=\mu_{f} H(0) .
\end{aligned}
$$

- Autocorrélation et densité spectrale

En développant l'expression de l'autocorrélation $R_{x x}(\tau)=E[x(t) x(t+\tau)]$ et en prenant la transformée de Fourier, on montre que la densité spectrale de la réponse s'écrit :

$$
S_{x x}(\omega)=|H(\omega)|^{2} S_{f f}(\omega) .
$$

- Exemple du système masse-ressort excité en bruit blanc.

Alors $S_{f f}(\omega)=S_{0}$. La moyenne quadratique de la réponse :

$$
\begin{aligned}
& E\left[x^{2}(t)\right]=R_{x x}(0)=\int_{-\infty}^{+\infty} S(\omega) d \omega= \\
& \int_{-\infty}^{+\infty}\left[\left(\omega_{0}^{2}-\omega^{2}\right)^{2}+4 a^{2} \omega_{0}^{2} \omega^{2}\right)^{-1} S_{0} d \omega=\left(\pi S_{0}\right) / 2 a \omega_{0}^{3} .
\end{aligned}
$$

\subsection{Systèmes à un nombre fini de degrés de liberté (figure 4)}

\subsubsection{Problème " unitaire " dans le temps}

Les notions précédentes se généralisent sous forme matricielle. Ainsi $h_{i j}(t)$ est la réponse $x_{i}(t)$ due à $f_{j}(t)=$ $\delta(t)$. La superposition donne alors :

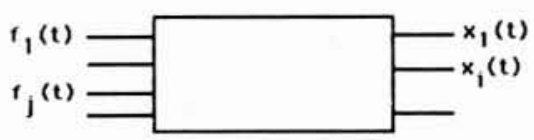

4. Système à nombre fini de degrés de liberté.

$$
\{x(t)\}=\int_{-\infty}^{+\infty}[h(\theta)]\{f(t-\theta)\} d \theta .
$$

\subsubsection{Problème " unitaire " en fréquence}

$H_{i j}(\omega) e^{i \omega t}$ est la réponse $x_{i}(t)$ due à $f_{j}(t)=e^{i \omega t}$. L'application du principe de superposition donne:

$$
\{x(t)\}=(2 \pi)^{-1} \int_{-\infty}^{+\infty} d \omega \int_{-\infty}^{+\infty}[H(\omega)]\{f(\tau)\} e^{i \omega(t-\tau)} d \tau .
$$

Les relations entre les transformées de Fourier sont :

$$
\begin{aligned}
{[h(t)]=(2 \pi)^{-1} \int_{-\infty}^{+\infty}[H(\omega)] e^{i \omega t} d \omega ; } \\
{[H(\omega)]=\int_{-\infty}^{+\infty}[h(t)] e^{i \omega t} d t . }
\end{aligned}
$$

\subsubsection{Statistiques de réponse}

La matrice des corrélations $\left[R_{X X}(\tau)\right]=$ $E\left[\{x(t)\}\{x(t+\tau)\}^{T}\right]$ est composée des éléments $R_{i j}(\tau)=$ $E\left[x_{i}(t) x_{j}(t+\tau)\right]$.

La matrice des densités spectrales $S_{x x}(\omega)$ est composée des éléments

$$
S_{i j}(\omega)=(2 \pi)^{-1} \int_{-\infty}^{+\infty} R_{i j}(\tau) e^{-i \omega \tau} d \tau
$$

La réponse matricielle des densités spectrales s'exprime en fonction de l'excitation sous la forme :

$$
\left[S_{x x}(\omega)\right]=[H(-\omega)]\left[S_{f f}(\omega)\right][H(\omega)]^{T} .
$$

\subsection{Systèmes continus}

\subsubsection{Réponse à une excitation appliquée en un point}

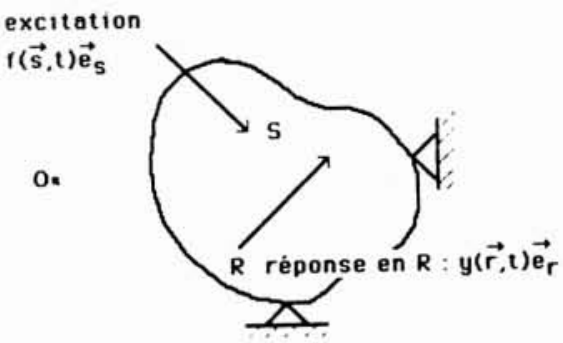

5. Excitation appliquée en un point.

Une excitation $f(\mathbf{s}, t)$ est appliquée dans la direction $\mathbf{e}_{s}$ au point $S$ repéré par le vecteur $\mathbf{s}=\mathrm{OS}$ (figure 5). La réponse en $R$ repéré par le vecteur $\mathbf{r}$ est $y(\mathbf{r}, t)$ dans une direction indiquée par le vecteur unitaire $\mathbf{e}_{r}$. 
Pour $f(\mathbf{s}, t)=\delta(t)$ la réponse en $R$ est $h(\mathbf{r}, \mathbf{s}, t)$ et pour $f(\mathbf{s}, t)=e^{i \omega t}$ la réponse est $H(\mathbf{r}, \mathbf{s}, \omega) e^{i \omega t}$, grandeurs liées par les relations de Fourier:

$$
\begin{gathered}
H(\mathbf{r}, \mathbf{s}, \omega)=\int_{-\infty}^{+\infty} h(\mathbf{r}, \mathbf{s}, t) e^{-i \omega t} d t, \\
h(\mathbf{r}, \mathbf{s}, t)=(2 \pi)^{-1} \int_{-\infty}^{+\infty} H(\mathbf{r}, \mathbf{s}, \omega) e^{i \omega t} d \omega .
\end{gathered}
$$

La relation entre les densités spectrales est tout à fait analogue à celle rencontrée précédemment :

$$
S_{y y}(\mathbf{r}, \omega)=H^{\star}(\mathbf{r}, \mathbf{s}, \omega) H(\mathbf{r}, \mathbf{s}, \omega) S_{f f}(\omega),
$$

où $H^{\star}$ est la quantité complexe conjuguée de $H$ (changement de $\omega$ en $-\omega)$.

\subsubsection{Réponse à une excitation répartie}

\subsubsection{Généralités}

L'excitation en chaque point $\mathbf{s}$ est alors caractérisée par un champ de pression $p(\mathbf{s}, t)$ et $f(\mathbf{s}, t)=p(\mathbf{s}, t) d \mathbf{s}$ où $d \mathbf{s}$ est un élément de surface en $S$.

Alors la DSP de la réponse croisée entre deux points $R_{1}$ et $R_{2}$ est :

$$
\begin{aligned}
& S_{y y}\left(\mathbf{r}_{1}, \mathbf{r}_{2}, \omega\right)= \\
& \quad \int_{\mathrm{V}} d s_{1} \int_{V} d s_{2} H^{\star}\left(\mathbf{r}_{1}, \mathbf{s}_{1}, \omega\right) H\left(\mathbf{r}_{2}, \mathbf{s}_{2}, \omega\right) S_{p p}\left(\mathbf{s}_{1}, \mathbf{s}_{2}, \omega\right),
\end{aligned}
$$

où $V$ désigne le domaine du système linéaire.

Imaginons que la structure mécanique soit soumise à des fluctuations de paroi dues à un fluide. On constate que la connaissance du spectre d'intercorrélation des fluctuations de pression de paroi, à savoir $S_{p p}\left(\mathbf{s}_{1}, \mathbf{s}_{2}, \omega\right)$ permet de déterminer les amplitudes des mouvements de la structure dès lors que sont connues les fonctions de transfert mécanique $H(\mathbf{r}, \mathbf{s}, \omega)$ de la structure.

Remarque: la relation précédente est utilisée sous une forme simplifiée lorsque l'excitation est localisée en un certain nombre de points $\mathbf{s}_{\mathbf{i}}$.

Alors :

$S_{y y}\left(\mathbf{r}_{1}, \mathbf{r}_{2}, \omega\right)=\Sigma_{i} \Sigma_{j} H^{\star}\left(\mathbf{r}_{1}, \mathbf{s}_{\mathbf{i}}, \omega\right) H\left(\mathbf{r}_{2}, \mathbf{s}_{\mathbf{j}}, \omega\right) S_{p p}\left(\mathbf{s}_{\mathbf{i}}, \mathbf{s}_{\mathbf{j}} \omega\right)$.

Si ces sollicitations sont non corrélées :

$$
S_{p p}\left(\mathbf{s}_{\mathbf{i}}, \mathbf{S}_{\mathbf{j}}, \omega\right)=S_{p p}\left(\mathbf{s}_{\mathbf{i}}, \omega\right)
$$

si $i=j$, spectre d'autocorrélation au point $\mathbf{s}_{\mathbf{i}} ; S_{p p}\left(\mathbf{s}_{\mathbf{i}}, \mathbf{S}_{\mathbf{j}}, \omega\right)$ $=0$ si $i \neq j$, alors

$S_{y y}\left(\mathbf{r}_{1}, \mathbf{r}_{2}, \omega\right)=\Sigma_{i} H^{*}\left(\mathbf{r}_{1}, \mathbf{s}_{\mathbf{i}}, \omega\right) H\left(\mathbf{r}_{2}, \mathbf{s}_{\mathbf{i}}, \omega\right) S_{p p}\left(\mathbf{s}_{\mathbf{i}}, \omega\right)$.
Le spectre d'autocorrélation de la réponse s'écrit simplement :

$S_{y y}(\mathbf{r}, \omega)=\Sigma_{i}\left|H\left(\mathbf{r}, \mathbf{s}_{\mathbf{i}}, \omega\right)\right|^{2} S_{p p}\left(\mathbf{s}_{\mathbf{i}}, \omega\right)$, c'est-à-dire que dans ce cas seul le module de la fonction de transfert est nécessaire.

\subsubsection{Expression dans la base modale}

La fonction de transfert entre deux points $R$ et $S$ d'une structure dissipative appartenant à la classe pour laquelle existent des modes normaux s'écrit : $H(\mathbf{r}, \mathbf{s}, \omega)=$ $\Sigma_{j} A_{j}(\omega) \psi_{j}(\mathbf{r}) \psi_{j}(\mathbf{s})$. Les $\psi_{j}(\mathbf{r})$ désignent les formes propres de la structure et les $A_{j}(\omega)$ désignent les admittances complexes des modes propres soit :

$$
A_{j}(\omega)=i \omega\left[\mu_{j}\left(\omega^{2}{ }_{j}-\omega^{2}\right)+2 i \alpha_{j} \omega_{j} \omega\right]^{-1}
$$

où $\omega_{j}, \mu_{j}, \alpha_{j}$ sont la pulsation propre, la masse généralisée et le paramètre d'amortissement du mode $j$. Les $\psi_{j}$ forment une base complète orthogonale pour les déformations de la structure et l'on a :

$$
\int_{V} \rho(\mathbf{s}) \psi_{j}(\mathbf{s}) \psi_{k}(\mathbf{s}) d \mathbf{s}=\mu_{j} \delta_{j k}
$$

où

$$
\delta_{j k}=0 \text { si } j \neq k \text { et } \delta_{j j}=1
$$

En utilisant cette relation pour développer $S_{y y}\left(\mathbf{r}_{1}, \mathbf{r}_{2}\right)$, le spectre de puissance de la réponse au point $R$ s'écrit :

$$
S_{y y}(\mathbf{r}, \omega)=\Sigma_{j} \Sigma_{k} \psi_{j}(\mathbf{r}) \psi_{k}(\mathbf{r}) A_{j}^{*}(\omega) A_{k}(\omega) Q_{j k}(\omega),
$$

où

$$
Q_{j k}(\omega)=\iint_{V} S_{p p}\left(\mathbf{s}_{1}, \mathbf{s}_{2}, \omega\right) \psi_{j}\left(\mathbf{s}_{1}\right) \psi_{k}\left(\mathbf{s}_{2}\right) d \mathbf{s}_{1} d \mathbf{s}_{2}
$$

est la densité interspectrale de puissance des forces généralisées $F_{j}(t)$ et $F_{k}(t)$ des modes $j$ et $k$ appliquées sur $V$ et telles que :

$$
F_{j}(t)=\int_{V} p(\mathbf{r}, t) \psi(\mathbf{r}) d \mathbf{r} .
$$

Ainsi :

$E\left[F_{j}(t) F_{k}(t+\tau)\right]=$

$\iint_{V} E\left[p\left(\mathbf{s}_{1}, t\right) p\left(\mathbf{s}_{2}, t+\tau\right)\right] \psi_{j}\left(\mathbf{s}_{1}\right) \psi_{k}\left(\mathbf{s}_{2}\right) d \mathbf{s}_{1} d \mathbf{s}_{2}$,

expression qui fait apparaître la fonction d'intercorrélation de la pression et dont la transformée de Fourier redonne $Q_{j k}(\psi)$.

Le spectre d'autocorrélation $S_{y y}(\mathbf{r}, \omega)$ s'écrit en définitive :

$$
\begin{aligned}
S_{y y}(\mathbf{r}, \omega)=\Sigma_{j}\left|A_{j}^{2}(\omega)\right| \psi_{j}^{2}(\mathbf{s}) & Q_{\mathrm{ss}}(\omega)+ \\
& \sum_{j \neq k} A_{j}^{*} A_{k} \psi_{j}(\mathbf{s}) \psi_{k}(\mathbf{s}) Q_{j k}(\psi)
\end{aligned}
$$

naires. De nombreuses questions (non stationnarité, non linéarités) ont été négligées: on pourra consulter la bibliographie. Quant aux aspects pratiques et concrets, ils seront abordés dans les exposés suivants.
L'exposé s'est limité à des considérations générales sur les systèmes linéaires soumis à des excitations station- 


\section{Bibliographie}

BOURGINE Alain : Sur une approche statistique de la dynamique vibratoire des structures. Publication ONERA No 149 (1973).

Crandall S.H.: Random vibration, MIT Press, Cambridge, Mass., 1963 .
KLyatskin V.I.: Ondes et équations stochastiques dans les milieux aléatoirement non homogènes. Editions de Physique, Les Ulis, 1985.

LIN Y.K. : Probabilistic theory of structural dynamics. Mc Graw Hill Book Company, 1967.

NEWLAND D.E. : An introduction to random vibration and spectral analysis. Longman Group Limited, England, 1984.

SvETLICKIJ V.A. : Vibrations aléatoires dans les systèmes mécaniques, Technique et Documentation. Paris 1980.

\author{
Adresses des auteurs \\ Monsieur A. Coubat \\ Laboratoire de mécanique appliquée \\ route de Gray \\ La Bouloie \\ 25030 Besançon Cedex \\ Tél. : (81) 53.81 .22 \\ Monsieur R.J. Gibert \\ CEA - CEN de Saclay \\ DEMT/SMTS \\ 91191 Gif-sur-Yvette Cedex \\ Tél. : 69.08.20.59
}

M. SAGNER. - Quelles sont les techniques d'analyse qui pourraient être utilisées pour l'étude des phénomènes nonlinéaires?

M. COUBAT. - Pour certaines catégories de problèmes, il est possible d'utiliser l'équation de Folker-Planck à condition de connaître la loi d'évolution du mouvement et aussi de pouvoir résoudre les équations correspondantes.

M. GIBERT. - La réponse aléatoire des structures nonlinéaires est complexe à prévoir et il n'existe pas pour cela de théorie générale. Il est d'ailleurs à remarquer que dans la pratique le caractère aléatoire de la réponse peut venir:

- soit du fait d'une instabilité non-linéaire de nature chaotique du système,

- soit d'une excitation aléatoire proprement dite.

Une possibilité est, en effet, de résoudre les équations de Folker-Planck, mais pour les systèmes industriels cela conduit à une formulation extrêmement lourde qui est due au grand nombre de degrés de liberté, et pour laquelle il n'existe pas d'outil numérique à l'heure actuelle.
Dans la pratique, les simulations numériques qui consistent à créer un processus source et à calculer la réponse du système au cours du temps sont couramment utilisées.

Pour obtenir une information correcte sur les grandeurs statistiques associées à la réponse, il faut effectuer plusieurs calculs temporels qui rendent la démarche assez lourde égale ment.

Une autre possibilité est de définir des modèles linéaires qui, dans les conditions d'excitations considérées, sont équivalents à la réponse du système non-linéaire selon des critères choisis.

L'utilisation de ces modèles linéaires équivalents nécessite bien évidemment une validation expérimentale ou numérique.

M. DAT. - En effet, il n'existe pas de méthodes générales pouvant résoudre les problèmes de non-linéarité qui soient industriellement utilisables. Même dans le cas des excitations harmoniques, ce problème reste délicat. L'expérience acquise montre que des méthodes sont particulièrement bien adaptées à certains types de non-linéarité et que pour d'autres nonlinéarités ce sont d'autres méthodes qu'il faut utiliser. 